\title{
Carnets
}

Revue électronique d'études françaises de l'APEF

Première Série - 4 Numéro Spécial | 2012

Invasions \& Évasions

\section{La France parmi nous: conscience historique et éducation européenne}

\section{Cristina Robalo Cordeiro}

\section{OpenEdition}

1 Journals

\section{Édition électronique}

URL : http://journals.openedition.org/carnets/8042

DOI : 10.4000/carnets.8042

ISSN : 1646-7698

Éditeur

APEF

Édition imprimée

Date de publication : 1 juin 2012

Pagination : 357- 364

Référence électronique

Cristina Robalo Cordeiro, «La France parmi nous: conscience historique et éducation européenne », Carnets [En ligne], Première Série - 4 Numéro Spécial | 2012, mis en ligne le 23 juin 2018, consulté le 20 avril 2019. URL : http://journals.openedition.org/carnets/8042 ; DOI : 10.4000/carnets.8042

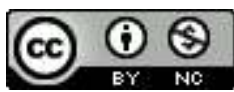

Carnets est mis à disposition selon les termes de la licence Creative Commons - Atribution - Pas d'utilisation commerciale 4.0 International. 


\title{
LA FRANCE PARMI NOUS : \\ Conscience historique et éducation européenne
}

CRISTINA ROBALO CORDEIRO

Université de Coimbra

crobalo@uc.pt

\begin{abstract}
Résumé
En conclusion d'un colloque réunissant historiens et littéraires, il est sans doute opportun, pour en mieux souligner l'intérêt, de dénoncer l'ambivalence épistémologique de sa problématique. Parlonsnous en effet du même objet quand le discours de la littérature et celui de l'histoire s'entrecroisent ? II convient de se souvenir que la première vise à mettre le lecteur en état d'hystérie, lui faisant vivre les événements qu'elle recrée, tandis que la seconde, se voulant connaissance scientifique, a le souci premier d'être objective, c'est-à-dire impassible. Une éducation européenne doit donner sa part à l'une et à l'autre, sans confondre leurs enseignements. Cette tâche pédagogique est notre devoir commun.
\end{abstract}

\begin{abstract}
As a conclusion to a colloquium which brought together historians and literature teachers, it is probably necessary, in order to stress its interest, to denounce its problematic's epistemological "ambivalence". Are we talking about the same object when the discourse of literature and the discourse of history are mingling their words? We must remember that the former aims at putting the reader in a state of hysteria, leading him to live the events it recreates, while the latter, aspiring to be a scientific knowledge, wants to be objective, i.e. impassible. A European education should give its place to both, without mixing their teachings. This pedagogical task is our common duty.
\end{abstract}

Mots-clés: littérature, hystérie, histoire, science, éducation, Europe.

Keywords: literature, hysteria, science, education, Europe. 
Comme la journée a été très longue, venant après celle d'hier, également très riche, vous autoriserez, vous encouragerez même l'ouvrière de la onzième heure que je suis à s'acquitter tambour battant de sa lourde tâche.

J'ai été investie par les organisateurs de l'honorable fonction de conclure ce colloque préparé de longue main et qui, semble-t-il, a pleinement atteint son objectif : réunir dans la bonne humeur, à l'occasion du deuxième centenaire des invasions de la Péninsule lbérique par les troupes de Napoléon, des historiens, des littéraires, des éducateurs autour de la cause française, telle qu'elle a été vécue, soutenue ou combattue des deux côtés de la frontière avant, pendant et après les temps héroïques qui ont servi de prétexte à notre rencontre.

L'idée ne manquait pas d'attraits. Le titre, le sous-titre, l'affiche, le programme du colloque, tous très réussis, annonçaient, en fanfare, un joyeux carnaval culturel où viendraient fraterniser non seulement les couleurs des trois nations mais aussi les démarches, les points de vue, les objets d'étude. On ne peut qu'applaudir à ce parti pris de réconciliation.

Après deux siècles - et tant d'autres horreurs - il est devenu possible de jouer avec les événements de 1810, de mêler la fiction aux faits, l'art d'écrire à l'art militaire, l'amour à la guerre. Toutes les permutations sont permises. Des invasions aux évasions, le renversement se fait très naturellement comme de l'histoire à la littérature, de la politique à la pédagogie, de la France arrogante à la France humiliée.

Nos rapports avec la France se placent sous le signe de l'alternance ou, plus justement, de l'ambivalence affective : tour à tour ou tout à la fois, on l'aime, on la déteste, elle nous charme et nous irrite, on l'admire, on la jalouse. Du Moyen Age à nos jours, il en a été ainsi. Si, dans l'ancienne Europe, l'Angleterre a été la plus vieille alliée, si l'Espagne a été la plus vieille ennemie, la France aura été la plus vieille maîtresse du Portugal. Ces contradictions passionnelles, ces fluctuations de l'âme se sont retrouvées dans les communications, où la francomanie et la francophobie se sont donné libre cours, chacune avec ses raisons et ses déraisons.

Si j'essaie de condenser ainsi la substance de cette rencontre, en en soulignant le caractère délibérément éclectique, voire composite - mais la vie n'est-elle pas elle-même un modèle de cacophonie ? - c'est que je voudrais essayer de remettre un peu d'ordre dans les perspectives comme il appartient à une "conférence de clôture ". Car autant le mélange des genres, des époques, des espaces, des démarches et des systèmes de référence a ajouté de vitalité à vos échanges, autant il risque d'ôter de rigueur épistémologique aux conclusions qu'il faut maintenant en dégager. 
Je vais faire en sorte qu'une apparence de logique ressorte de toutes les notions et de toutes les émotions qui ont été brassées durant ces deux jours et dont on a bien voulu me communiquer l'essentiel.

Une telle logique ne peut s'obtenir qu'à condition de sacrifier d'abord ce qui, précisément, a fait l'originalité des débats: ces interférences, ces croisements, ces recoupements entre des lignes qu'il faut considérer pourtant dans leur distinction si on tient à comprendre de quoi l'on parle. Selon qu'on aborde la thématique principale - les invasions françaises - en historien ou en littéraire, la lumière change, la chaleur varie, le souci d'objectivité augmente ou diminue.

A mon sens, une des questions fortes posées par ce colloque franco-ibérique, où se sont amicalement côtoyés, entre autres spécialistes, des littéraires et des historiens, revient à se demander ce qui précisément distingue la littérature de l'histoire. C'est là un sujet qui a déjà fait couler beaucoup d'encre dans l'univers et dans cet amphithéâtre même. II me semble important d'y revenir quand on s'interroge sur la représentation des invasions françaises et, plus généralement, sur l'influence de la France dans nos climats.

Une remarque préalable s'impose cependant: le thème des invasions françaises tend de nombreux pièges aux amis de la France. Par quelle conversion soudaine de valeurs psychologiques va-t-on, le temps d'un colloque, prendre en horreur l'objet de notre affection constante et maudire le pays que l'on tient pour une seconde patrie ? Ce partage, ce conflit est le mien comme, sans doute, celui de la plupart d'entre vous. Aurais-je tendu la main au général Masséna si j'avais fais alors partie du Bataillon Académique? Aurais-je "collaboré "avec l'Occupant ? Peut-être ces questions, relevant de l'illusion rétrospective qui nous fait mettre le présent dans le passé, n'ont-elles de sens que pour l'imagination littéraire. La rigueur de l'historiographie les réprouve.

Pour rendre hommage au côté français de ma formation, je vous propose donc un plan, non pas de bataille certes mais de dissertation, une sorte de schéma tripartite où vous pourrez apercevoir, à votre guise, une thèse - le parti de la littérature - une antithèse - le parti de l'histoire - et une synthèse, que je vous laisse deviner d'après le titre que j'ai donné à mon propos. Et rassurez-vous, ces trois moments seront de plus en plus courts !

Je commence donc par définir la position de la littérature devant le passé, et, en l'occurrence, face aux horreurs des guerres péninsulaires.

Pour ce faire il m'est utile de me référer à un passage de la première des Cinq leçons de psychanalyse, où Freud caractérise l'hystérie en affirmant : "les hystériques souffrent de réminiscences. Leurs symptômes sont les résidus et les symboles de certains événements traumatiques. Symboles commémoratifs, à vrai dire. "(Freud, 1977 : 15). Le comportement hystérique, qu'il décrit ensuite dans une comparaison très parlante, n'est pas sans analogie avec celui du lecteur de romans historiques en personne : 
A Londres, non loin du London Bridge, vous remarquez une colonne moderne très haute que l'on appelle 'The Monument'. Elle doit rappeler le souvenir du grand incendie qui, en 1666, éclata tout près de là et détruisit une grande partie de la ville. C'est un symbole commémoratif. Mais que diriez-vous d'un habitant de Londres qui se mettrait à pleurer devant 'The Monument' la destruction de la ville de ses pères, alors que cette ville est depuis longtemps renée de ses cendres et brille aujourd'hui d'un éclat plus vif encore que jadis ? (id. Ibid.)

Imaginez seulement que nous ne soyons pas à Londres, mais à Madrid, sur la Praça del Sol, lieu d'une célèbre insurrection, ou à Saragosse, dont la moitié de la population fut exterminée en février 1809, ou encore à Leiria, sur le site de l'exécution en masse du 5 juin 1808. Qui verserait aujourd'hui des larmes devant les monuments commémoratifs de ces terribles épisodes? Personne à vrai dire, sauf le lecteur du roman qui les revivrait sur un mode hystérique comme une «résurrection de la vie intégrale "du passé.

Cette dernière formule pourrait être de Freud mais elle est de Michelet, le grand historien romantique qui voulait en effet que l'histoire soit une vision directe du passé (voir la Préface de 1869 à son Histoire de France). Mais, avec tout son génie, il se trompait de genre. C'est à la littérature, non à l'histoire, que revient une telle restitution fantasmatique d'événements enfouis dans les profondeurs du temps collectif. C'est la magie du roman, non de l'étude historique, de nous faire éprouver des passions anciennes, de ranimer les flammes mortes.

La vraie machine à remonter le temps, ce n'est pas l'histoire mais la littérature. Ainsi fait Balzac dans la nouvelle intitulée "El Verdugo "dont l'action se situe, durant les guerres péninsulaires, dans une petite ville espagnole de la côte méditerranéenne : à la suite d'une conspiration éventée "in extremis", toute une famille d'aristocrates va périr sous la hache tenue par le fils cadet selon l'ordre donné par le général français et avant que la population entière ne soit passée par les armes. Le but de Balzac n'est pas de nous faire seulement connaître un épisode atroce mais de nous le faire revivre. Et c'est très différent comme vous le verrez en lisant le conte.

En somme, si la littérature nous purifie de nos passions c'est qu'elle procède, comme la psychanalyse, sur un mode cathartique. Je ne crains donc pas, sous l'incantation des romanciers, de me replonger hystériquement ou, ce qui revient à peu près au même, "romantiquement», dans l'époque des invasions françaises. Je sais que la littérature m'inocule le mal mais qu'elle m'en guérit presque aussitôt. Le vrai sang a depuis longtemps séché sur les places et dans les champs. Après huit ou neuf générations, la souffrance, le deuil ont disparu, remplacés par une quantité d'autres douleurs. Seuls quelques statues ou 
bas-reliefs mutilés attestent encore la barbarie de ces troupes fanatisées. Comme Londres, nos villes dévastées ont été reconstruites et les passions partisanes ou patriotiques n'ont plus de feu que dans les romans ou dans les films.

C'est pourquoi j'ai parlé de "jeu " tout à l'heure. Nous pouvons esthétiquement jouer, comme la jolie affiche du colloque, avec les invasions françaises alors que des drames plus proches de nous restent intouchables. Et de fait ni les Ambassades ni les Consulats de France n'ont été mis à sac cette année par des Portugais ou des Espagnols ivres de vengeance. Nous avons bien d'autres motifs de colère!

Je viens de désigner la place et la fonction de la littérature, tendanciellement fictionnelle même quand elle s'inspire de faits ou de documents authentiques. A ce titre, elle est toujours tragique sans être jamais sérieuse. Un bon tiers des communications en ont fait la démonstration.

Le point de vue de l'historien est tout autre. II ne cherche ni à faire pleurer ni à faire rire mais à faire comprendre, comme Spinoza le disait de la philosophie. Laissant toutes les passions à la porte de son cabinet, il peut quantifier la souffrance en calculant le nombre des victimes, mais il ne cherchera pas à la transmettre. Si l'objectivité en histoire est une limite idéale, c'est aussi pour l'historien une obligation, obligation à laquelle aucun romancier, même réaliste, ni aucun journaliste, au contact immédiat avec l'événement ou avec l'opinion, ne se soumet. Plusieurs exposés nous ont montré sur des exemples précis que la presse balance entre les deux pôles de l'histoire et de la littérature.

C'est dire que le dialogue des historiens avec les littéraires devient difficile, peut-être impossible dès qu'il s'approfondit - à moins que l'on ne fasse des premiers les psychanalystes des seconds et vice versa! Et c'est aussi souligner l'incongruité scientifique mais également l'intérêt expérimental d'un colloque réunissant les deux espèces comme si on voulait rassembler dans un même congrès des chimistes et des alchimistes. J'insiste sur le fait que je définis des lignes virtuelles, la littérature et l'histoire pouvant, bien entendu, dans la pratique, comme la science et la poésie, nouer entre elles les alliances les plus monstrueuses, c'est-à-dire les plus intéressantes.

Grâce à l'histoire, nous saisissons mieux l'enjeu, du côté français, et je ne dis pas l'excuse des invasions successives de la Péninsule. L'ennemi véritable, c'était l'Anglais. Et avec les armées de Napoléon c'est la Révolution française qui se répand au-delà des Pyrénées comme elle l'a fait dans le reste de l'Europe en trouvant partout en face d'elle, visible ou invisible, le bras de la "perfide Albion ».

Dès 1798, Jean-François Bourgoing, ancien ambassadeur de la République à Madrid, s'étonnait, comme par avance, de ces Portugais qui "affectionnent leurs tyrans "- les Moines et les Anglais - et "repoussent leurs libérateurs ", les Français il va sans dire: "C'est pour d'avides étrangers que le sol du Portugal se féconde. Ses ateliers ont brisés ou 
languissent, et il s'épuise pour aviver ceux de Manchester et de Birmingham "(Bourgoing, Voyage du ci-devant duc du Châtelet en Portugal, Buisson, Paris, 1798, t. 1, p.VII).

Mais cet intellectuel jacobin ne comprenait pas que la liberté se conquiert plutôt qu'elle ne s'impose. Une des rares lois morales à laquelle l'histoire moderne semble obéir, c'est que la liberté prend son temps mais qu'elle finit toujours par triompher. II est connu que c'est dans la Péninsule Ibérique que Napoléon a pour la première fois éprouvé pour son malheur la force irrésistible de la volonté des peuples.

Si l'histoire peut nous apprendre autre chose, liée à la première, c'est que la résistance à l'oppresseur, même très puissant, n'est jamais vaine. Cette leçon est ressortie de la brillante étude de notre collègue Ana Cristina Araújo et peut nous encourager dans notre effort présent contre tous les ennemis plus ou moins occultes de la liberté et que dénonçait hier matin avec éloquence Marc Fumaroli.

C'est sur cette leçon que je m'appuierai pour esquisser, en quelques notes éparses, ma dernière partie qui, vous l'avez pressenti, ne peut porter que sur l'éducation et tout l'espoir que nous devons y mettre.

"Education européenne": quelques-uns parmi vous ont peut-être lu le livre de Romain Gary qui porte ce titre : écrit en 1943 par un combattant de la France libre, il évoque la résistance polonaise. C'est l'histoire d'un misérable petit maquis, tenaillé par la faim et l'hiver, à peine capable de commettre de temps à autre un piètre attentat contre les troupes allemandes. Mais cette poignée de malheureux réfractaires sauve l'honneur de leur pays. Parmi eux se trouve un jeune romancier qui soutient leur fragile moral par ses récits et ses rêves, comme si la littérature et la résistance n'étaient pas séparables.

L'Europe hitlérienne a duré moins de cinq ans. L'Europe napoléonienne n'a pas tenu dix ans. La nôtre, mécontente d'elle-même comme elle est, a l'avantage d'être la nôtre, démocratiquement constituée sinon gouvernée et, espérons-le, plus durable. Tout le monde néanmoins s'accorde pour reconnaître qu'il manque une âme et un cœur à cette Europe de la paix. Pour l'en pourvoir, un immense travail culturel est à faire, dans les écoles et les universités. II faut saluer, à cet égard, la publication d'ouvrages comme L'Histoire de l'Europe, écrite par douze historiens européens, ou Le Vocabulaire européen des Philosophies, ouvrages qui ne sont pourtant que des prototypes de l'œuvre pédagogique à construire.

Car pour que l'Europe ne soit pas un concept politique vide, ou une simple administration au service d'on ne sait quels intérêts, il importe que toutes les nations qui la composent prennent une nouvelle conscience d'elles-mêmes dans leurs relations avec les autres, principalement à travers la connaissance de leurs histoires et la jouissance de leurs littératures, de mieux en mieux traduites. 
Cette nouvelle conscience ne naîtra pas sans l'engagement individuel des millions d'enseignants que compte l'Europe unie. Encore faut-il que nous y croyions nous-mêmes, à cette Europe. Encore faut-il que nous sachions, laissant loin derrière nous les guerres et les invasions fratricides, célébrer, de temps en temps, à l'image de la France réconciliée de juillet 1790, la fête de la fédération. Ce colloque européen nous aura au moins permis, dans le froid de novembre et dans des circonstances générales très adverses, de vivre, au croisement de l'histoire et de la littérature, un petit moment d'évasion. Une petite fête de la culture et de l'amitié. 


\section{Bibliographie}

FREUD, S. (1974). Cinq leçons de psychanalyse. Paris : Petite bibliothèque Payot. MICHELET, J. (1977). Histoire de France. Edition de Paul Viallaneix. Paris : Flammarion. BOURGOING, Jean-François. (1798). Voyage du ci-devant duc du Châtelet en Portugal. an VI de la République, T. I. Paris : F. Buisson. 